\title{
Optical polarimetry toward the Pipe nebula: revealing the importance of the magnetic field ${ }^{\star}$
}

\author{
F. O. Alves ${ }^{1}$, G. A. P. Franco ${ }^{2}$, and J. M. Girart ${ }^{1}$ \\ 1 Institut de Ciències de l'Espai (CSIC-IEEC), Campus UAB, Facultat de Ciències, C5 par 2a , 08193, Bellaterra, Catalunya, Spain \\ e-mail: [oliveira;girart]@ieec.uab.es \\ 2 Departamento de Física - ICEx - UFMG, Caixa Postal 702, 30.123-970 Belo Horizonte, Brazil \\ e-mail: franco@fisica.ufmg.br
}

Received 29 April 2008 / Accepted 3 June 2008

\section{ABSTRACT}

\begin{abstract}
Context. Magnetic fields are proposed to play an important role in the formation and support of self-gravitating clouds and the formation and evolution of protostars in such clouds.

Aims. We attempt to understand more precisely how the Pipe nebula is affected by the magnetic field.

Methods. We use $R$-band linear polarimetry collected for about 12000 stars in 46 fields with lines of sight toward the Pipe nebula to investigate the properties of the polarization across this dark cloud complex.

Results. Mean polarization vectors show that the magnetic field is locally perpendicular to the large filamentary structure of the Pipe nebula (the "stem"), indicating that the global collapse may have been driven by ambipolar diffusion. The polarization properties clearly change along the Pipe nebula. The northwestern end of the nebula (B59 region) is found to have a low degree of polarization and high dispersion in polarization position angle, while at the other extreme of the cloud (the "bowl") we found mean degrees of polarization as high as $\approx 15 \%$ and a low dispersion in polarization position angle. The plane of the sky magnetic field strength was estimated to vary from about $17 \mu \mathrm{G}$ in the $\mathrm{B} 59$ region to about $65 \mu \mathrm{G}$ in the bowl.

Conclusions. We propose that three distinct regions exist, which may be related to different evolutionary stages of the cloud; this idea is supported by both the polarization properties across the Pipe and the estimated mass-to-flux ratio that varies between approximately super-critical toward the B59 region and sub-critical inside the bowl. The three regions that we identify are: the B59 region, which is currently forming stars; the stem, which appears to be at an earlier stage of star formation where material has been through a collapsing phase but not yet given birth to stars; and the bowl, which represents the earliest stage of the cloud in which the collapsing phase and cloud fragmentation has already started.
\end{abstract}

Key words. ISM: clouds - ISM: individual objects: Pipe nebula - ISM: magnetic fields - techniques: polarimetric

\section{Introduction}

Understanding the role that magnetic fields play in the evolution of interstellar molecular clouds is one of the outstanding challenges of modern astrophysics. One problem related to star formation concerns the competition between magnetic and turbulent forces. The prevailing scenario of how stars form is quasi-static evolution of a strongly magnetized core into a protostar following influence between gravitational and magnetic forces. By ambipolar diffusion, i.e., the drift of neutral matter with respect to plasma and magnetic field, gravity finds a way to overcome magnetic pressure and eventually win the battle (e.g., Mestel \& Spitzer 1956; Nakano 1979; Mouschovias \& Paleologou 1981; Lizano \& Shu 1989). However, doubts about the validity of this theory were expressed because of the apparent inconsistency between the expected and inferred lifetimes of molecular clouds. This inconsistency inspired some researchers to propose a new theory in which star formation is driven by turbulent supersonic flows in the interstellar medium. Magnetic fields may be present in this theory, but they are too weak to be energetically important (e.g. Elmegreen \& Scalo 2004; Mac Low \& Klessen 2004). It must be noted, however, that some

^ Based on observations collected at Observatório do Pico dos Dias, operated by Laboratório Nacional de Astrofísica (LNA/MCT, Brazil). results (Tassis \& Mouschovias 2004; Mouschovias et al. 2006) demonstrate that the ambipolar-diffusion-controlled star formation theory is not in contradiction with molecular cloud lifetimes and star formation timescales.

Previous optical polarimetric observations toward wellknown forming molecular clouds have enabled the large-scale magnetic field associated with these regions to be studied (e.g. Goodman et al. 1990). In this work, we introduce the general results of a polarimetric survey conducted for the Pipe nebula, a nearby (130-160 pc, Lombardi et al. 2006; Alves \& Franco 2007) and massive $\left(10^{4} M_{\odot}\right)$ dark cloud complex that appears to provide a suitable laboratory for investigating magneto-turbulent phenomena. The Pipe nebula exhibits little evidence of star formation activity despite having an appropriate mass. Until now, the only confirmed star-forming region in this nebula was B59 (Brooke et al. 2007), an irregularly-shaped dark cloud located at the northwestern end of the large filamentary structure that extends from $(l, b) \approx\left(0^{\circ}, 4^{\circ}\right)$ to $(l, b) \approx\left(357^{\circ}, 7^{\circ}\right)$. This apparently low efficiency in forming stars may be an indication of youth. Alves et al. (2007) identified, in this cloud, 159 cores of effective diameters between 0.1 and $0.4 \mathrm{pc}$, and estimated masses ranging from 0.5 to $28 M_{\odot}$, supposedly in a very early stage of development. A further investigation of these cores (Lada et al. 2008) discovered that most of them appeared to be pressure 


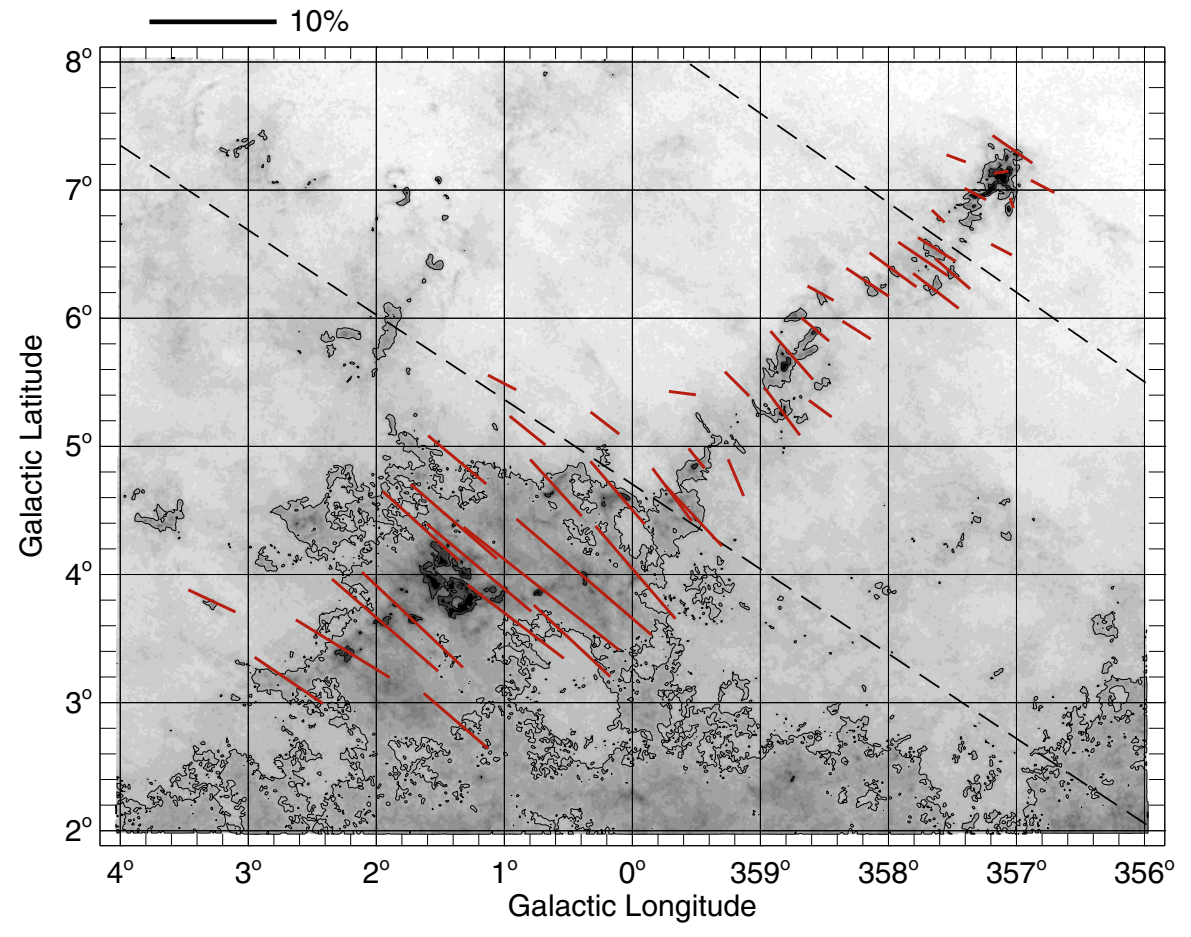

Fig. 1. Mean polarization vectors, for each of the observed 46 fields, overplotted on the dust extinction map of the Pipe nebula obtained by Lombardi et al. (2006). The lengths of these vectors are proportional to the scale indicated in the top left-hand corner. Only stars showing $P / \sigma_{P} \geq 10$ were used in the calculus of the mean polarization and position angle. The dashed-lines indicate the celestial meridians defined by $17^{\mathrm{h}} 14^{\mathrm{m}} 30^{\mathrm{s}} .0$ and $17^{\mathrm{h}} 27^{\mathrm{m}} 40^{\varsigma} .0$ (see text and Fig. 2). confined and in equilibrium with the surrounding environment, and that the most massive $\left(\gtrsim 2 M_{\odot}\right)$ cores were gravitationally bound. They suggested that the measured dispersion in internal core pressure of about a factor of $2-3$ could be caused by either local variations in the external pressure, or the presence of internal static magnetic fields with strengths of less than $16 \mu \mathrm{G}$, or a combination of both. The results derived from our optical polarimetric observations indicate that the magnetic field probably plays a far more important role in the Pipe nebula.

\section{Observations}

The polarimetric data were acquired using the $1.6 \mathrm{~m}$ and the IAG $60 \mathrm{~cm}$ telescopes of Observatório do Pico dos Dias (LNA/MCT, Brazil) during observing runs completed between 2005 to 2007. These data were acquired by using a CCD camera specially adapted to allow polarimetric measurements; for a full description of the polarimeter see Magalhães et al. (1996). $R$-band linear polarimetry, by means of deep CCD imaging, was obtained for 46 fields, each with a field of view of about $12^{\prime} \times 12^{\prime}$, distributed over more than $7^{\circ}$ (17 pc in projection) covering the main body of the Pipe nebula. The reference direction of the polarizer was determined by observing polarized standard stars. For all observing seasons, the instrumental position angles were perfectly correlated with standard values. The survey contains polarimetric data of about 12000 stars, almost 6600 of which have $P / \sigma_{P} \geq 10$. The results presented in this Letter are based on the analysis of the latter group of stars. Details of observations, data reduction, and the analysis of the small-scale polarization properties within each observed area, will be described in a forthcoming paper (Franco et al. 2008).

\section{Polarization at the Pipe nebula}

To analyze the polarization pattern in the Pipe nebula, we estimated the mean polarization and position angle for each observed field. To improve the precision of the mean values, we selected those objects with $P / \sigma_{P} \geq 10$ and observed polarization angle $\theta_{\text {obs }}$ within the interval $\left(\theta_{\mathrm{av}}-2 \sigma_{\mathrm{std}}\right) \leq \theta_{\mathrm{obs}} \leq\left(\theta_{\mathrm{av}}+2 \sigma_{\mathrm{std}}\right)$ where, $\theta_{\text {av }}$ and $\sigma_{\text {std }}$ are the mean polarization angle and standard deviation of each field sample, respectively. We then estimated the mean Stokes parameters for each field, from the individual values for each star weighted by the estimated observational error. Most fields show a distribution of polarization position angles that resembles a normal distribution, although a more complex distribution is evident in some directions. A detailed analysis of these distributions is beyond the scope of the present Letter and will be presented in the aforementioned paper.

Figure 1 shows the mean polarization vectors overlaid on the 2MASS infrared extinction map of the Pipe nebula derived by Lombardi et al. (2006). For most fields, the values of the mean polarization and position angle were obtained from samples of more than 100 stars. The high signal-to-noise ratio of our data set ensures good statistics in our analyses and implies that the degree of polarization measured for most fields and, in particular, the significant range of mean polarization values derived along the Pipe (from 1 to $15 \%$ ) are truly remarkable. It is also remarkable that the polarization position angle does not change significantly along the $17 \mathrm{pc}$ extent of the Pipe nebula covered by our observations $\left(\langle\theta\rangle \simeq 160^{\circ}-10^{\circ}\right.$ for 37 of the 46 fields, where the mean position angles are given in equatorial coordinates, measured from north to east). Although the physical processes involved in grain alignment is a debated issue (see Lazarian 2003, for a comprehensive review on this subject), it is widely believed that starlight polarization is caused by the alignment of elongated dust grains by the magnetic field, as suggested by the pioneering work of Davis \& Greenstein (1951). Based on this assumption, the polarization map showed in Fig. 1 provides an outline of the magnetic field component parallel to the plane of the sky. The almost perpendicular alignment between the magnetic field and the main axis of the Pipe's stem is clearly evident.

It is instructive to analyze the behavior of polarization properties along the Pipe nebula: the left panels of Fig. 2 present the distribution of the mean polarization and the polarization angle 

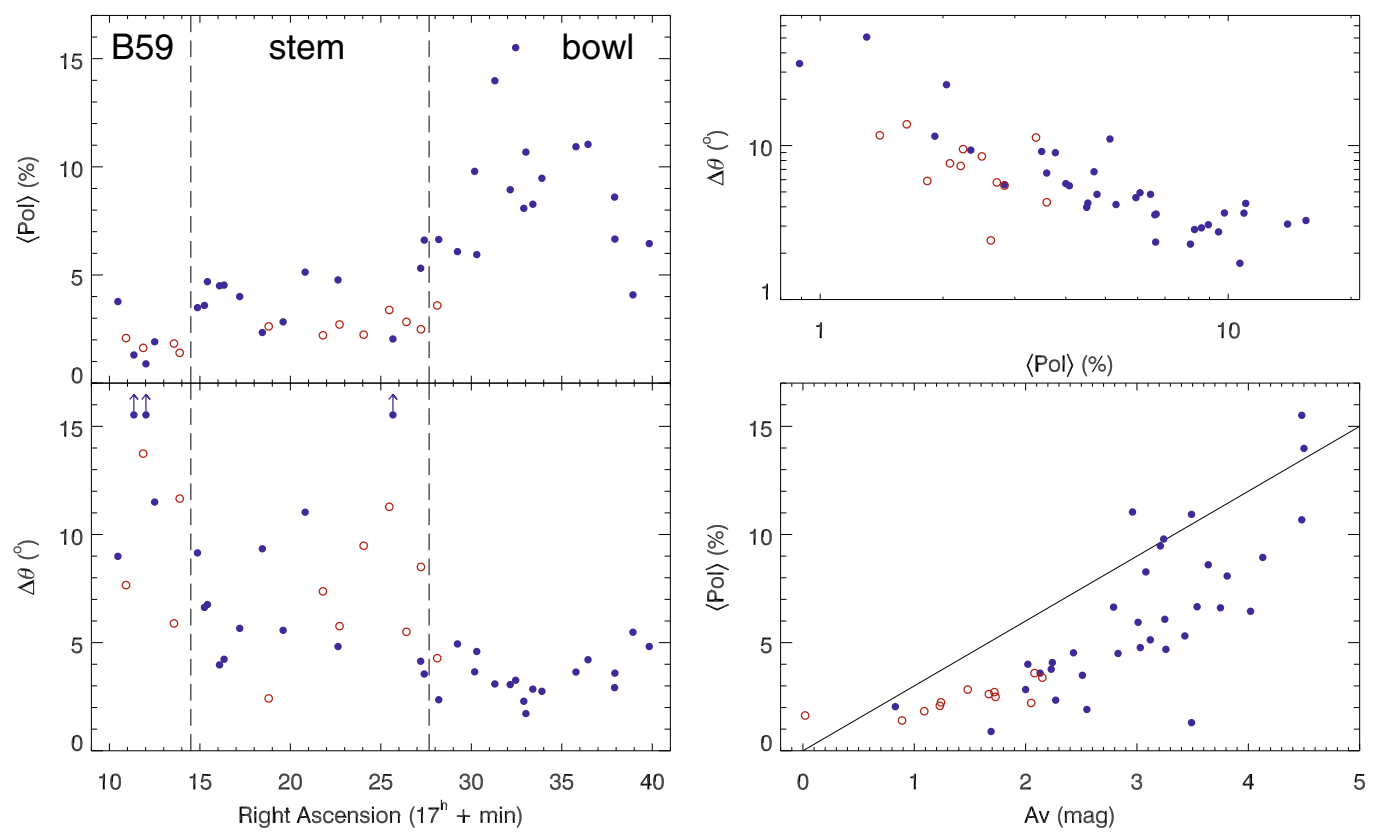

Fig. 2. Left panels: distribution of the mean polarization and of the polarization angle dispersion, $\Delta \theta$, as a function of the right ascension of the observed areas, respectively. The polarization angle dispersion is corrected by its mean error (i.e., $\Delta \theta^{2}=\sigma_{\text {std }}^{2}-\left\langle\sigma_{\theta}\right\rangle^{2}$ ). The vertical dashedlines delimits the transition between regions with different polarimetric properties. Filled and open dots represent values for fields with and without associated dense cores, respectively. As shown by the botton right panel, the regions traced by the optical polarimetry have extinction of $A_{\mathrm{V}} \lesssim 2.2 \mathrm{mag}$ for fields without cores, while the ones associated with cores show $0.8 \lessgtr A_{\mathrm{V}} \lesssim 4.5 \mathrm{mag}$. Top right panel: correlation between dispersion in polarization angle and mean polarization. Botton right panel: mean polarization versus visual absorption derived from the $2 \mathrm{MASS}$ data for the observed stars with $P / \sigma_{P} \geq 10$. The solid line represents optimum alignment efficience $\left(P(\%)=3 \times A_{\mathrm{V}}\right)$.

dispersion as a function of the right ascension of the observed areas, which runs almost parallel to the main axis of the Pipe's stem. Since the polarization properties of each field are inhomogeneous, a global analysis allows one to distinguish three regions throughout the cloud with rather different features between them. These regions, separated by dashed-lines in Figs. 1 and 2, can be identified as: the B59 region, at the northwestern end of the cloud; the main filamentary structure (the stem of the Pipe); and the irregular-shaped gas at the other extreme end (the "bowl"). We note that fields without cores (open dots in Fig. 2) show a smaller variation in polarization properties than fields with cores (filled dots).

The lowest mean polarizations are observed in the vicinity of B59, the only place in the Pipe with evidence of star formation. Seven out of eight observed fields in this region show mean polarization degrees of around 1-2\%. This region has a large polarization angle dispersion. Indeed, two fields have a dispersion in polarization angles $\Delta \theta \gtrsim 25^{\circ}$ - these are indicated in the bottom right panel of Fig. 2 by the arrowed dots - and show the lowest mean degree of polarization among the observed fields. We point out that the field showing the highest dispersion in polarization angles $\left(\Delta \theta \simeq 51^{\circ}\right)$ has a line of sight passing close to the densest core of B59, the most opaque region of the Pipe (Román-Zúñiga et al. 2007), and that our sample has only 12 stars for which $P / \sigma_{P} \geq 10$.

Toward the stem region the mean polarization rises a few percent and the polarization angle dispersion decreases slightly with respect to B59. Most fields containing dense cores show a mean polarization degree $(\simeq 3-5 \%)$ that is higher than fields without cores $(\simeq 2-3 \%)$. However, this difference is unclear from the position angle dispersion values, which show a large range of values for both types of field $\left(\Delta \theta \simeq 3^{\circ}-12^{\circ}\right)$.
The bowl has a significantly different mean polarization and dispersion in position angles: for this region, we measure the highest degree of polarization and the lowest dispersion in position angles. Most observed fields in the bowl shows a mean polarization higher than about $8 \%$ (up to $15 \%$ ) and a dispersion in polarization angles of less than $5^{\circ}$. This part of the cloud has the most precise alignment between the mean polarization vectors of neighboring fields. The high polarization degree in the bowl is unusual, since the polarization degree of this type of dark interstellar clouds is typically 1 order of magnitude lower than we measure and rarely reaches such high values (e.g., Vrba et al. 1993; Whittet et al. 1994, 2001, for optical polarimetric data on $\rho$ Oph, Chamaeleon I, and Taurus dark clouds, respectively). Such a result implies a high efficiency of grain alignment for the interstellar dust in those fields, and that the magnetic field in the bowl is aligned close to the plane of the sky (otherwise the efficiency would be even higher).

Figure 2 (top right panel) also indicates the distribution of $\Delta \theta$ as a function of the mean polarization: it is a clear observational fact for the observed fields that the higher the mean polarization, the lower the dispersion in polarization angles. The anticorrelation between the dispersion in polarization angles and polarization degree has a similar dependence for fields with and without cores. This anti-correlation could be due just to projection effects: the magnetic field direction changes along the Pipe nebula. However, this scenario would imply a polarization efficiency and a magnetic field strength (see below) that would be unusually high over the entire nebula. The star formation activity in B59 probably precludes this scenario.

What can the aforementioned polarization properties tell us about the magnetic field in the Pipe nebula? Our dispersion in polarization angles can be used to estimate the magnetic field strength for the observed fields from the modified 
Chandrasekhar-Fermi formula (Chandrasekhar \& Fermi 1953; Ostriker et al. 2001). The volume density and line width of the molecular line emission associated with the dust that produces the observed optical polarization and extinction can be estimated from the molecular data available in the literature. Thus, extrapolating the median volume density of cores given by Lada et al. (2008) to the optical polarization zone (which is typically at a distance of about $5^{\prime}-0.2 \mathrm{pc}$ in projection - from the center of the cores), we obtain a volume density of $n\left(\mathrm{H}_{2}\right) \simeq 3 \times 10^{3} \mathrm{~cm}^{-3}$. We also adopt the line width found for $\mathrm{C}^{18} \mathrm{O}$ toward the cores in B59 and the stem, $0.4 \mathrm{~km} \mathrm{~s}^{-1}$, and the bowl, $0.5 \mathrm{~km} \mathrm{~s}^{-1}$ (the values used here are the ones given by Muench et al. 2007). Assuming these values, we find that the magnetic field strength in the B59 region, stem, and bowl, in the plane of the sky, are about 17,30 , and $65 \mu \mathrm{G}$, respectively (the uncertainty in the values are probably less than a factor of 2). Adopting a mean visual extinction of $3 \mathrm{mag}$ for the molecular cloud traced by the optical polarimetry, we find that the mass-to-flux ratio is about 1.4 (slightly super-critical) for B59, in contrast to 0.8 and 0.4 (subcritical) for the stem and the bowl, respectively.

The almost perpendicular alignment between the magnetic field and the main axis of the Pipe nebula's stem indicates clearly that this part of the cloud contracted in the direction of the field lines. This agrees with predictions of the ambipolar-diffusion driven model, for which the first evolutionary stage of a typical cloud is dynamical relaxation along field lines, almost without lateral contraction, until a quasi-equilibrium state is reached (e.g., Fiedler \& Mouschovias 1993; Tassis \& Mouschovias 2007). Indeed, the magnetic pressure $\left(P_{\text {mag }}=B^{2} / 8 \pi\right)$ of the diffuse part of the cloud (where is most of the mass) is the dominant source of pressure in the direction perpendicular to the field lines $\left(12 \times 10^{5}\right.$ and $2.6 \times 10^{5} \mathrm{~K} \mathrm{~cm}^{-3}$ for the bowl and stem, respectively), being higher than the pressure due to the weight of the cloud $\left(P_{\text {cloud }} / k=10^{5} \mathrm{~K} \mathrm{~cm}^{-3}\right.$, according to Lada et al. 2008). This can explain the clear elongated structure perpendicular to the magnetic field of the whole nebula.

The derived mean polarization degree and dispersion in polarization angles are consistent with a scenario in which the B59 region, the stem, and the bowl are experiencing different stages of their evolution. The weak magnetic field derived for the B59's neighboring appears to be the reason for it being the only known active star-forming site in the cloud. Following the evolutionary sequence, the stem with a mass-to-flux ratio close to unity would be the part of the cloud in a transient evolutionary state, which is experiencing ambipolar diffusion but has not yet given birth to stars. Finally, the high polarization degree of the bowl combined with the low dispersion in the mean polarization vectors implies that the magnetic field in this part of the cloud has a major role in regulating the collapse of the cloud material compared to the other parts. This would imply that the bowl is in a primordial evolutionary state (in the sub-critical regime), not yet flattened neither elongated. However, the presence of multiple and clearly evident cores implies that fragmentation is already occurring inside the bowl. A similar case, in a more evolved state, appears to be the Taurus molecular cloud complex (Nakamura \& Li 2008).

\section{Conclusions}

We have described the global polarimetric properties of the Pipe nebula as an increasing polarization degree along the filamentary structure from B59 towards the bowl, while the dispersion in polarization angles decreases along this way. Our results appears to indicate that there exist three regions in the Pipe nebula of distinct evolutionary stages: since the mean orientation angle of the mean polarization vectors is perpendicular to the longer axis of the cloud, this implies that the cloud collapse is taking place along the magnetic field lines. We can subdivide the Pipe nebula into the following components:

- B59, the only active star-forming site in the cloud. For the observed fields, we measure a large dispersion in polarization angle and low polarization degree.

- The stem, which collapsed by means of ambipolar diffusion but has not yet given birth to stars. It appears to represent a transient evolutionary state between B59 and the bowl.

- The bowl, which contains the fields of the highest values of mean polarization and the lowest values of dispersion in polarization angle. These values imply that the dust grains in the bowl are highly aligned by a rather strong magnetic field. For this reason, the bowl may represent the start of the contraction phase during a very early evolutionary stage.

Acknowledgements. We thank the staff of the Observatório do Pico dos Dias (LNA/MCT, Brazil) for their hospitality and invaluable help during our observing runs. Drs. A. M. Magalhães and A. Pereyra are acknowledged for providing the polarimetric unit and the software used for data reductions. We made use of NASA's Astrophysics Data System (NASA/ADS) and of data products from the Two Micron All Sky Survey. This research has been partially supported by CEX APQ-1130-5.01/07 (FAPEMIG, Brazil) and AYA2005-08523-C03 (Ministerio de Ciencia e Innovación, Spain).

\section{References}

Alves, F. O., \& Franco, G. A. P. 2007, A\&A, 470, 597

Alves, J., Lombardi, M., \& Lada, C. J. 2007, A\&A, 462, L17

Brooke, T. Y., Huard, T. L., Bourke, T. L., et al. 2007, ApJ, 655, 364

Chandrasekhar, S., \& Fermi, E. 1953, ApJ, 118, 113

Davis, L. J., \& Greenstein, J. L. 1951, ApJ, 114, 206

Elmegreen, B. G., \& Scalo, J. 2004, ARA\&A, 42, 211

Fiedler, R. A., \& Mouschovias, T. C. 1993, ApJ, 415, 680

Franco, G. A. P., Alves, F. O., \& Girart, J. M. 2008, in preparation

Goodman, A. A., Bastien, P., Menard, F., \& Myers, P. C. 1990, ApJ, 359, 363

Lada, C. J., Muench, A. A., Rathborne, J., Alves, J. F., \& Lombardi, M. 2008, ApJ, 672, 410

Lazarian, A. 2003, J. Quantitative Spectrosc. Radiat. Transf., 79, 881

Lizano, S., \& Shu, F. H. 1989, ApJ, 342, 834

Lombardi, M., Alves, J., \& Lada, C. J. 2006, A\&A, 454, 781

Mac Low, M.-M., \& Klessen, R. S. 2004, Rev. Mod. Phys., 76, 125

Magalhães, A. M., Rodrigues, C. V., Margoniner, V. E., Pereyra, A., \& Heathcote, S. 1996, in Polarimetry of the Interstellar Medium, ASP Conf. Ser., 97, 118

Mestel, L., \& Spitzer, Jr., L. 1956, MNRAS, 116, 503

Mouschovias, T. C., \& Paleologou, E. V. 1981, ApJ, 246, 48

Mouschovias, T. C., Tassis, K., \& Kunz, M. W. 2006, ApJ, 646, 1043

Muench, A. A., Lada, C. J., Rathborne, J. M., Alves, J. F., \& Lombardi, M. 2007, ApJ, 671, 1820

Nakamura, F., \& Li, Z.-Y. 2008 [arXiv:0804 . 4201v1]

Nakano, T. 1979, PASJ, 31, 697

Ostriker, E. C., Stone, J. M., \& Gammie, C. F. 2001, ApJ, 546, 980

Román-Zúñiga, C. G., Lada, C. J., Muench, A., \& Alves, J. F. 2007, ApJ, 664, 357

Tassis, K., \& Mouschovias, T. C. 2004, ApJ, 616, 238

Tassis, K., \& Mouschovias, T. C. 2007, ApJ, 660, 388

Vrba, F. J., Coyne, G. V., \& Tapia, S. 1993, AJ, 105, 1010

Whittet, D. C. B., Gerakines, P. A., Carkner, A. L., et al. 1994, MNRAS, 268, 1 Whittet, D. C. B., Gerakines, P. A., Hough, J. H., \& Shenoy, S. S. 2001, ApJ, 547, 872 